\title{
Research Progress in Ferroptosis for Digestive Carcinoma
}

\author{
Bin Jiang and XiangYan Liu* \\ Department of Thoracic Surgery, Shandong University, China
}

*Corresponding author: XiangYan Liu, Department of Thoracic Surgery, Shandong Provincial Hospital, Shandong University, China.

Received Date: October 28, 2019

Published Date: November 12, 2019

\section{Introduction}

Cell death plays a significant role in the survival and development of individual. It includes necrosis and apoptosis, which are the two most common forms of cell death. As more and more researches progress, some findings can't be explained by apoptosis. Nowadays, some researchers have found that some antitumor drugs developed against apoptosis exist apoptosis escape and chemotherapy tolerance. So, is there any other form of cell death to overcome the resistance of tumor cells? Camptothecin (CPT) can induce apoptotic cell death, which is characterized by nuclear pyknosis and karyorhexis. Dolma et al. [1] found no similar morphological changes in erastin-treated cells. Therefore, erastininduced cell death is considered a nonapoptotic cell death. Soon afterwards, Yang et al. [2] and Yagoda et al. [3] found that this form of cell death can be suppressed by iron chelators, accompanied by an increase in intracellular reactive oxygen species. In 2012, Dixion et al. [4] named this new form of cell death as ferroptosis, which is characterized by ferric ion involved and is different from apoptosis, necrosis and autophagy. The discovery of ferroptosis provides an imaginative space for the treatment of tumors and overcoming drug resistance, opens new avenues for the development of new drugs. This review summarizes the research progress of ferroptosis in digestive system tumors.

\section{Result}

\section{Gastric carcinoma (GC)}

By means of westernblot, RT-qPCT and immunohistochemical (IHC), Sun et al. [5] found overexpressing perilipin2 (PLIN2) in gastric carcinoma cell lines accompanied by the expression of PRDM11, ACSL3, LC3A, ALOX15 descended while the expression of IPO7 increased. When PLIN2 was knocked down, the above situation is reversed. Furthermore, PLIN2 is also verified to alter lipid metabolism in gastric carcinoma. Given ferroptosis contain changes of above gene and lipid metabolism, the study considered that PLIN2 inhibited ferroptosis in gastric carcinoma. Hao et al. [6] certified that downregulating Cysteine Dioxygenase 1 (CDO1) expression is conducive to the elevation of GSH levels and decrease of ROS levels simultaneously, at last facilitating ferroptosis resistance and tumor growth. The study also found that inhibition of c-Myb weakens erastin-induced ferroptosis. In addition, overexpression of c-Myb upregulates glutathione peroxidase 4 (GPX4, an antioxidant enzyme) expression. Hence, c-Myb may mediate ferroptosis together with CD01, but the specific mechanism is still unclear. It is reported by Niu et al. [7] that Physcion 8-0- $\beta$-glucopyranoside (PG) can up-regulate the expression of glutaminase 2 (GLS2). Upregulating miR-103a-3p targets GLS2 to weaken PG-induced ferroptosis. PG activates ferroptosis in gastric carcinoma cells as well as downregulates the suppressive effect of miR-103a-3p on GLS2 expression. Furthermore, GLS2 targets p53 gene, which exerts antineoplastic effect by inducing ferroptosis [8].

\section{Hepatocellular carcinoma (HCC)}

Previous studies on ferroptosis in the HCC process have focused on System XC- and Gpx4-related signaling pathways. Lately, $\mathrm{Ou}$ et al. [9] engineered a low-density lipoprotein nanoparticle reconstituted with the natural $\omega-3$ PUFA, docosahexaenoic acid (LDL-DHA). It can selectively kill HCC cells and slow down the growth of hepatocellular tumors in vivo. After ingesting LDL-DHA nanoparticles, the intracellular lipid ROS will increase, while GSH and GPX4 will decrease. They supposed LDL-DHA inhabits HCC via ferroptosis. This was later verified by ferrostatin (fer-1), a specific inhibitor of ferroptosis. Nanoparticle drugs provide a new direction for ferroptosis in cancer cells. Hepatocyte nuclear factor 4 alpha (HNF4A) is important to liver development [10] and is up-regulated in HCC $[11,12]$. Hypermethylated in cancer 1 (HIC1) restrained tumor cells growth [13]. Zhang et al. [14] discovered targets related 
to ferroptosis are regulated by HNF4A and HIC1 in HCC. HIC1 and HNF4A restrict each other in HCC. HIC1 identified as stimulating ferroptosis while HNF4A has been identified as inhibitor of ferroptosis in HCC. Activation ferroptosis destroyed the balance between HNF4A and HIC1. HNF4A-mediated anti-ferroptosis is more powerful than that of HIC1-mediated pro-ferroptosis. To destroy the equilibrium between HIC1 and HNF4A might be useful to inducing ferroptosis in HCC. Sun et al. [15] confirmed that the p62-Keap1-NRF2 pathway modulates the sensibility of HCC cells towards ferroptosis by controlling the expression of NRF2. Bai et al. [16] confirmed that silencing Sigma-1 receptor (S1R) enhanced the effect of anticancer drug sorafenib in HCC cells both in vitro and in vivo. This phenomenon was regulated by GPX4 variation and ROS accumulation. S1R and NRF2 have similar functions in HCC cells treated with sorafenib.

\section{Pancreatic carcinoma (PC)}

Xie et al. [17] demonstrated that CCT137690(an aurora kinase inhibitor) is capable of inducing pancreatic ductal adenocarcinoma cancer (PDAC) cell death, such as apoptosis, necroptosis, and ferroptosis. But only necroptosis signaling played a dominant role. After a short while, Yamaguchi et al. [18] found that Piperlongumine (PL) can induce the death of PC cells via ferroptosis. According to their results, PL-induced ferroptosis was enhanced by Cotylenin A (CN-A) and/or SSZ (a clinical drug and ferroptosis inducer). The triple therapy with PL, CN-A and SSZ is very effective against PC.

\section{Colorectal carcinoma (CRC)}

Wei et al. [19] concluded that D13(a synthesized compound) downregulates the expression of GPX4 and induced the accumulation of lipid peroxidation, resulting in ferroptosis in CRC cells. Guo et al. [20] demonstrated that cisplatin was able to induce both ferroptosis and apoptosis in CRC cells, moreover, the depletion of GSH and concomitant invalidation of GPXs played an important role in the potential mechanism. What's more, duplex therapy of cisplatin and erastin showed significant enhancement in their antitumor effect.

\section{Conclusion and Perspective}

As is well-known that patients with digestive system cancer have a very poor 5-year survival rate. Previous studies have shown that ferroptosis may be an important method to treat carcinoma. Recent researches in ferroptosis for digestive system tumors has achieved very significant achievement. Some molecules participate in ferroptosis in digestive system carcinoma, including PLIN2, CD01, c-Myb, PG, GLS2, miR-103a-3p, HNF4A, HIC1, NRF2, S1R and CN-A. A few drugs and chemical synthesis, for example, LDLDHA, SSZ, D13 and cisplatin also play a crucial part in ferroptosis. In addition, signaling pathways such as p62-Keap1-NRF2 and miR103a-3p/GLS2 axis have also attracted people's attention. However, the study of ferroptosis in esophageal cancer has not been reported. The corresponding mechanism in esophageal carcinoma still needs to be investigated. Finally, ferroptosis is indeed an important breakthrough in the treatment of cancer, while clarifying the specific molecular mechanism of ferroptosis in digestive tumors still requires a lot of work.

\section{Acknowledgements}

None.

\section{Conflict of Interest}

No conflict of interest.

\section{References}

1. Dolma S, Lessnick SL, Hahn WC, Stockwell BR (2003) Identification of genotype-selective antitumor agents using synthetic lethal chemical screening in engineered human tumor cells. Cancer Cell 3(3): 285-296.

2. Yang WS, Stockwell BR (2008) Synthetic Lethal Screening Identifies Compounds Activating Iron-Dependent, Nonapoptotic Cell Death in Oncogenic-RAS-Harboring Cancer Cells. Chemistry \& Biology 15(3): 234-245.

3. Yagoda N, von Rechenberg M, Zaganjor E, Bauer AJ, Yang WS, et al. (2007) RAS-RAF-MEK-dependent oxidative cell death involving voltagedependent anion channels. NATURE 447(7146): 865-869.

4. Dixon SJ, Lemberg KM, Lamprecht MR, Skouta R, Zaitsev EM, et al. (2012) Ferroptosis: An Iron-Dependent Form of Nonapoptotic Cell Death. Cell 149(5): 1060-1072.

5. Sun X, Yang S, Feng X, Zheng Y, Zhou J, et al. (2019) The modification of ferroptosis and abnormal lipometabolism through overexpression and knockdown of potential prognostic biomarker perilipin2 in gastric carcinoma. Gastric Cancer.

6. Hao S, Yu J, He W, Huang Q Zhao Y, et al. (2017) Cysteine Dioxygenase 1 Mediates Erastin-Induced Ferroptosis in Human Gastric Cancer Cells. Neoplasia 19(12): 1022-1032.

7. Niu Y, Zhang JP, Tong YL, Jiansheng Li, Bingrong Liu, et al. (2019) Physcion 8-0- $\beta$-glucopyranoside induced ferroptosis via regulating miR103a-3p/ GLS2 axis in gastric cancer. Life Sciences.

8. R Kang, G Kroemer, D Tang (2019) The tumor suppressor protein p53 and the ferroptosis network. Free Radical Biol Med 133: 162-168.

9. Ou W, Mulik RS, Anwar A, McDonald JG, He X, et al. (2017) Low-density lipoprotein docosahexaenoic acid nanoparticles induce ferroptotic cell death in hepatocellular carcinoma. Free Radical Biology \& Medicine 112(1): 597-607.

10. Parviz F, Matullo C, Garrison WD, Savatski L, Adamson JW, et al. (2003) Hepatocyte nuclear factor 4alpha controls the development of a hepatic epithelium and liver morphogenesis. Nat Genet 34 (3): 292-296.

11. Xu L, Hui L, Wang S, Gong J, Jin Y, et al. (2001) Expression profiling suggested a regulatory role of liver-enriched transcription factors in human hepatocellular carcinoma. Cancer Res 61(7): 3176-3181.

12. Dill MT, Tornillo L, Fritzius T, Terracciano L, Semela D, et al. (2013) Constitutive Notch2 signaling induces hepatic tumors in mice. Hepatology 57(4): 1607-1619.

13. Ubaid Ullah, Andrabi SBA, Tripathi SK, Dirasantha O, Kanduri K, et al. (2018) Transcriptional repressor HIC1 contributes to suppressive function of human induced regulatory T cells. Cell Rep 22: 2094-2106.

14. Zhang X, Du L, Qiao Y, Zhang X, Zheng W, et al. (2019) Ferroptosis is governed by differential regulation of transcription in liver cancer Redox Biology 24: 2213-2317.

15. Sun X, Ou Z, Chen R, Niu X, Chen D, et al. (2016) Activation of the p62Keap1-NRF2 path- way protects against ferroptosis in hepatocellular carcinoma cells. Hepatology 63: 173-184.

16. Tao Bai, Pengxu Lei, Hao Zhou, Ruopeng Liang, Rongtao Zhu, et al. (2019) Sigmareceptor protects against ferroptosis in hepatocellular carcinoma cells. Journal of Cellular and Molecular Medicine Pp. 7349-7359.

17. Xie Y, Zhu S, Zhong M, Yang M, Sun X, et al. (2017) Inhibition of Aurora Kinase A Induces Necroptosis in Pancreatic Carcinoma. Gastroenterology 153(5): 1429-1443. 
18. Yamaguchi Y, Kasukabe T, Kumakura S (2018) Piperlongumine rapidly induces the death of human pancreatic cancer cells mainly through the induction of ferroptosis. International Journal of Oncology 52(3):10111022.

19. Wei G, Sun J, Hou Z, Luan W, Wang S, et al. Novel antitumor compound optimized from natural saponin albiziabioside a induced caspase- dependent apoptosis and ferroptosis as a p53 activator through the mitochondrial pathway. European Journal of Medicinal Chemistry 157: 759-772.

20. Guo J, Xu B, Han Q, Zhou H, Xia Y, et al. (2017) Ferroptosis: A Novel AntiTumor Action for Cisplatin. Cancer Research and Treatment 50(2): 445460. 